\title{
Installed Performance Assessment of a Boundary Layer Ingesting Distributed Propulsion System at Design Point
}

\author{
C. Goldberg*, D. Nalianda†, D. MacManus ${ }^{\ddagger}$ and P. Pilidis ${ }^{\S}$ \\ Cranfield University, Bedfordshire, MK43 OAL, United Kingdom \\ and \\ J. Felder \\ NASA Glenn Research Centre, Cleveland, Ohio, USA
}

\begin{abstract}
Boundary layer ingesting systems have been proposed as a concept with great potential for reducing the fuel consumption of conventional propulsion systems and the overall drag of an aircraft. These studies have indicated that if the aerodynamic and efficiency losses were minimised, the propulsion system demonstrated substantial power consumption benefits in comparison to equivalent propulsion systems operating in freestream flow. Previously assessed analytical methods for BLI simulation have been from an uninstalled perspective. This research will present the formulation of an rapid analytical method for preliminary design studies which evaluates the installed performance of a boundary layer ingesting system. The method uses boundary layer theory and one dimensional gas dynamics to assess the performance of an integrated system.

The method was applied to a case study of the distributed propulsor array of a blended wing body aircraft. There was particular focus on assessment how local flow characteristics influence the performance of individual propulsors and the propulsion system as a whole. The application of the model show that the spanwise flow variation has a significant impact on the performance of the array as a whole. A clear optimum design point is identified which minimises the power consumption for an array with a fixed configuration and net propulsive force requirement. In addition, the sensitivity of the system to distortion related losses is determined and a point is identified where a conventional free-stream propulsor is the lower power option. Power saving coefficient for the configurations considered is estimated to lie in the region of $15 \%$.
\end{abstract}

\section{Nomenclature}

$\begin{array}{ll}\text { Acronyms } & \\ A R & =\text { Aspect ratio } \\ B L & =\text { Boundary layer } \\ B L C & =\text { Boundary layer control } \\ B L I & =\text { Blended wing Body } \\ B W B & =\text { Fan pressure ratio } \\ F P R & =\text { Free-stream } \\ F S & =\text { Kinetic energy } \\ K E & =\text { Kinetic energy non-dimensional group } \\ K E G & \end{array}$

${ }^{*}$ PhD Researcher, Propulsion Engineering Centre, Cranfield University, Student Member.

${ }^{\dagger}$ Lecturer, Propulsion Engineering Centre, Cranfield University.

${ }^{\ddagger}$ Senior Lecturer, Propulsion Engineering Centre, Cranfield University, Senior Member.

$\S$ Head of Centre, Propulsion Engineering Centre, Cranfield University.

ฯ Aerospace Engineer, LTA, NASA Glenn Research Center, 21000 Brookpark Rd, Cleveland, OH 44145, Senior Member. 


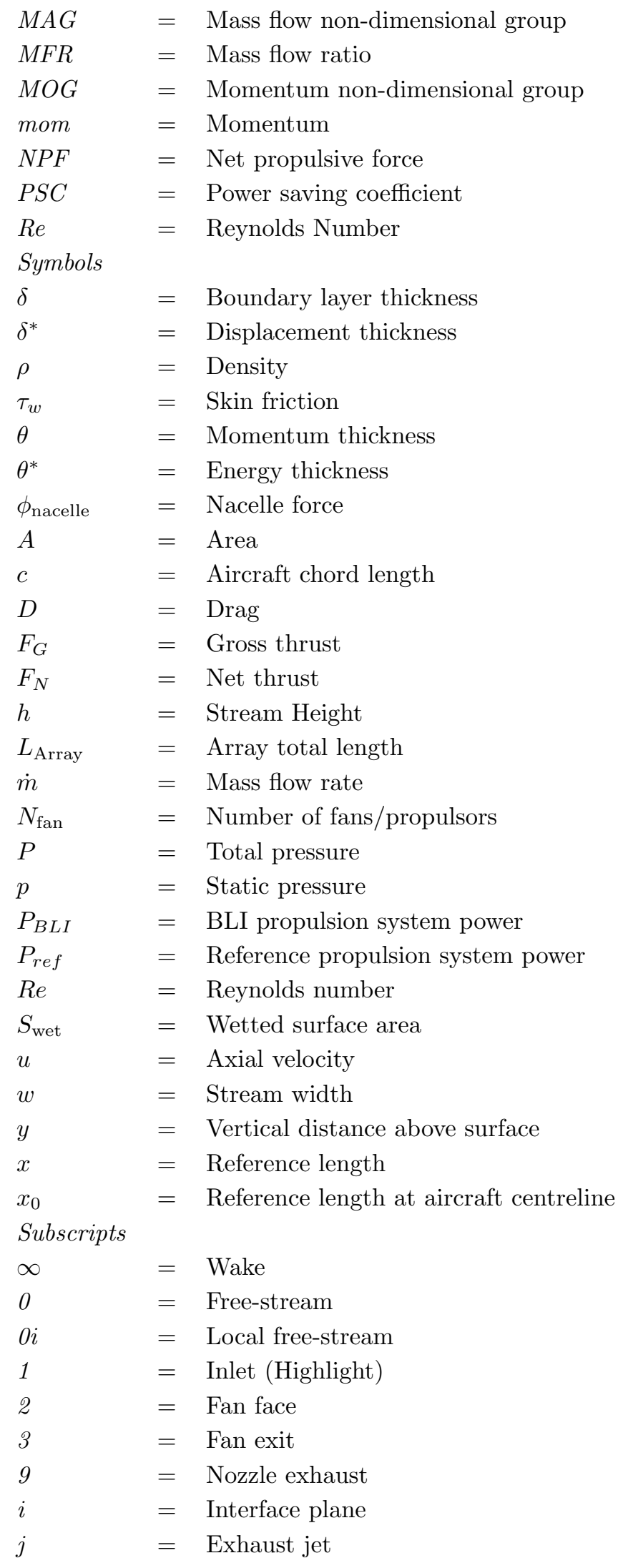

\section{Introduction}

In general, the boundary layer is considered to be detrimental to the performance of a conventional aircraft, as it contributes to drag and results in a momentum deficit, or wake. In the propulsion system, 
an ingested turbulent boundary layer can give rise to total pressure and swirl non-uniformities. which can have a negative impact on the performance of an engine. To avoid such problems with the quality of the ingested flow, the typical commercial aircraft planform is powered by podded engines. These are positioned to ingest free-stream air without any influence from the aircraft wake or boundary layer. Aircraft with more closely integrated engines, such as military configurations, often include some form of boundary layer bleed or diversion which modifies the boundary layer before it enters the inlet. As momentum deficit is one of the sources of aircraft drag, it follows that technologies to control or reduce the impact of the boundary layer are a potential route to an improvement in aircraft performance. Lower aircraft drag corresponds to lower thrust requirements, and hence has the potential to reduce the fuel consumption of the aircraft. The continuous increase in environmental awareness, coupled with the volatility of fuel price, means that reductions in fuel consumption are one of the primary drivers of aircraft development. As such, boundary layer control is one of the possible technological routes that can be taken during the design of an aircraft. Passive management of the boundary layer induced drag on an aircraft can be obtained through modification of the micro-scale nature of the aircraft surface. An option that may be applied without an extensive aircraft redesign is the application of sharkskin paint, which reduces turbulence perpendicular to the flow along the airframe [1]. Alternatively, highly smooth coatings on the surface of the aircraft can be used to reduce the airframe skin friction coefficient [2].

The concept of boundary layer control is not a new one. Early research suggested that the application of constant suction on the aircraft surface could reduce the drag of the aircraft through removal of the boundary layer [3]. The boundary layer also has the potential to be of benefit to the propulsion system through the application of boundary layer ingestion (BLI). BLI can re-energise the wake of the aircraft by accelerating ingested boundary layer flow back up to the free stream velocity. Such concepts have been utilised in marine propulsion, and can equally be considered for aviation applications [4]. By ingesting the boundary layer, less power is used by the propulsion system than for an propulsion system that produces the same thrust with free-stream air [5]. Additionally, wake re-energisation reduces the overall drag of the aircraft. However, ingestion of the boundary layer is not entirely beneficial to a propulsion system. The boundary layer is an inherently distorted flow, which will almost certainly have some impact on the performance of the system. The engine may be subject to distorted flow from the bottom to the top of the inlet, or radially along the fan blade, such as in the case of a propulsive fuselage [6]. Significant distortion can negate the power or fuel consumption benefits of a BLI system, such that a free-stream system is the more efficient option [5]. The levels of distortion can be exacerbated in more complicated inlet geometries, such as an S-duct intake. Boundary layers are prone to separation in an adverse pressure gradient, which is compounded by the development of classical secondary flows associated with the turning through an S-duct bend [7, 8]. Nonetheless, S-ducts have been considered as an option for a BLI system, as they support the application of embedded engines to reduce the profile of the aircraft. The use of passive, active or hybrid boundary layer flow control (BLC) can contribute towards a significant reduction in the distortion in intakes such as an S-duct [7]. BLI can be combined with a distributed propulsion system, in order to ingest a greater portion of the aircraft boundary layer. Aircraft such as NASA's N3-X make use of a distributed propulsor array mounted on the upper surface of a blended wing body (BWB) fuselage [10]. The high-aspect ratio spanwise or 'mail-slot' inlet allows for the ingestion of a significant proportion of the boundary layer. Similarly, the Silent Aircraft Initiative's SAX-40 makes use of three boundary layer ingesting engine clusters [11] which ingest $16.6 \%$ of the airframe boundary layer [5]. On conventional tube-and-wing configurations an aft mounted BLI propulsion system in the tail section of the airframe may be used to re-energise the fuselage wake $[6,12]$.

A problem arises when performance predictions of a BLI propulsion system are required. Evaluation of the typical podded engines on a conventional transport aircraft is a well-established method. The method allows for a relatively simple thrust and drag accounting between the aircraft and its engines. However, this distinction is a greater challenge for a BLI propulsion system. Flow that enters a BLI propulsion system has travelled over the aircraft surface and the separation of the airframe and propulsion system is therefore more complicated. This integration also influences performance calculations, as local flow characteristics are linked with the propulsion system location and the aircraft configuration. Previous research on the simulation of BLI systems can be broadly split into two categories: computational fluid dynamic (CFD) analyses of the problem as a whole, and lower-order analytical methods which evaluate the system using boundary layer and propulsion system performance theory. CFD methods are useful for the analysis of the combined aircraft and propulsion system. However, it is important to be able to predict the performance of the propulsion system at a preliminary design stage without expensive experimental methods or complex and time-consuming simulations. This is especially important during the evaluation of a set of competing technologies or for the process of configuration down-selection. It 
is therefore useful to have a rapid, low-order analytical method which is suitable for simulations of the overall performance of an air vehicle with an integrated BLI system.

There are a number of factors that should ideally be represented in a BLI simulation. The boundary layer has a deficit in mass flow, momentum and kinetic energy relative to free-stream flow. This deficit is due to a variation in pressure, velocity and density from the no-slip surface of the boundary layer. The distorted flow may also result in a reduction in fan or compressor efficiency and an increased total pressure loss in the intake. Evaluation of the influence of the boundary layer on performance is therefore a key aspect of a BLI model. The boundary layer itself may be modelled with simple correlations, integral boundary layer equations or a case-specific CFD analysis. These representations have been used in internal force control volumes to estimate the performance of the propulsion system [5, 13, 14]. These control volumes typically deal with the propulsion system as an isolated or uninstalled system in a similar manner to conventional propulsion system analysis. A challenge of the integral boundary layer or one-dimensional control volume method is that the influence of the boundary layer on fan or compressor performance is not directly represented. This may instead by estimated by a parallel compressor / parallel stream method [5, 15]. The method splits the flow into a number of streams to assist in prediction of asymmetric flow's impact on a compressor [16]. A more in-depth representation of an aircraft with boundary layer ingestion can be performed based on the energy of the system, as shown by Drela [17]. More recently, this has been expanded upon with an exergy approach by Arntz et al [18]. Representations of the energy of the combined aircraft and propulsion system avoid the challenge of thrust and drag definitions in an integrated system. However, they are complex methods that move away from the conventional force representations used in propulsion system models. In addition, the energy methods rely on knowledge of the configuration as a whole by the use of a control volume which encompasses the whole aircraft. This is difficult for propulsion system designers that work independently from airframe designers, or at the preliminary design stage. As with a full CFD analysis, the rapid analysis of multiple configurations with a full system energy analysis is a time-consuming activity. The combination of these factors limits the use of the method at a preliminary design stage. In addition, the method has limited application in aircraft performance models.

The previous methods for BLI simulation have drawbacks in that they rely either too little on the aircraft configuration (in the case of the uninstalled performance methods), or too much on the configuration (in the case of the exergy and energy methods). Nonetheless, conventional force control volume methods are more suitable for the purposes of both preliminary design and integration in aircraft performance analyses. Previous force control volume methods use a thrust and drag accounting system similar to the uninstalled performance calculations for conventional podded engines. However, BLI systems are inherently integrated and must include aspects of the aircraft configuration to sufficiently represent performance. The integration factor in a BLI system is considered to be a vital part of the system simulation. This work therefore builds on previous force control volumes to attempt to address this particular and key aspect of the technology assessment. The aim of this research is to develop a novel method that includes the integration aspects of the system, whilst remaining flexible enough to accommodate design changes or to be used for different configurations, on a consistent basis.

\section{A. Scope}

A key aspect of this research is to establish a method and work flow to simulate the performance of a propulsion system that ingests the boundary layer of the aircraft. The method aims to serve as a rapid low-order system for use in the preliminary design stage of a conceptual BLI aircraft. A primary component of the procedure is the simulation of integrated system, rather than the uninstalled performance typically used in previous analytical methods. This necessitates an unambiguous definition of the split between the airframe and propulsion system. A key factor of the integrated simulation is assessment of the influence of the airframe on the propulsion system and vice versa. This includes representation of how local flow characteristics (such as boundary layer thickness or local free-stream velocity) influence the propulsion system performance and how the wake re-energisation of a BLI system will influence the propulsion system requirements. The method aims to act as a module that can be integrated within conventional propulsion system performance methods.

The method was applied to the N3-X aircraft as a case study. The N3-X is a NASA conceptual blended wing body aircraft with a distributed BLI propulsor array [10]. This research first presents the theory behind the simulation method, followed by a description of the relevant formulae that are combined to create the simulation. The formulae employed represent one potential route to the boundary layer characteristics required for the performance simulation procedure. However, equivalent rapid methods 
may also be considered as an alternative, provided that the required flow characteristics are produced. A validation of the procedure for estimation of the boundary layer flow characteristics was performed against previous research. Finally, the influence of the aircraft and propulsion system configuration on the propulsion system performance was assessed. The simulation results include an assessment of how local flow characteristics influence performance, as a key component of the propulsion system simulation. Boundary layer characteristics are a function of the aircraft configuration, hence propulsors at different locations on the airframe operate with different inlet conditions. Gladin et al show that, for the BWB case considered, there is a possible $4 \%$ difference in specific fuel consumption of an engine at inboard and outboard locations on the airframe [19]. This difference in specific fuel consumption is induced by a change in Reynolds number and hence in boundary layer thickness and characteristics between engines in different locations. The results of the N3-X analysis serve to address performance aspects specific to the aircraft configuration and identify what factors may influence an optimum configuration.

\section{Theory}

\section{A. Thrust and Drag Accounting}

The forces produced by an aircraft in flight can be split to belong to either a propulsion system or an airframe force accounting system in support of conventional descriptions of aircraft performance [20]. In steady level flight, the net thrust produced by a propulsion system should be adequate to counter the drag of the combined airframe and installed engine. To calculate this net thrust parameter, it is necessary to account for both the gross thrust and the momentum drag of the system. Differentiating these forces is a relatively simple matter for an aircraft with podded engines, as there is limited interaction between the engine and airframe. However, for aircraft with more integrated architectures it becomes more difficult to differentiate between the the airframe and the propulsion system [21]. This is typically the case in a military aircraft, where the engines are often embedded in the airframe. However, it is nonetheless useful to separate the force accounting of the engine and airframe, as the design of the two components will generally not be combined and will be performed by different groups at a preliminary design stage.

A number of accounting systems exist that define thrust and drag from different positions (or stations) relative to the engine and its nacelle. Engine thrust is typically quoted as a 'standard net thrust' term $\left(F_{N}\right)$, the difference between the gross thrust at the nozzle exit $\left(F_{G 9}\right)$ and the gross thrust far upstream $\left(F_{G 0}\right)$. Such a definition of thrust can be evaluated independently of flight conditions and is ideal for uninstalled thrust tests of an engine [20].

$$
F_{G}=\dot{m} u+A\left(p-p_{0}\right)
$$

Net thrust is the common definition for the thrust produced by an aircraft, however, in an integrated system the installation terms are also linked to the performance. The integration aspects of the system should therefore be included as a part of the force accounting for greater accuracy. In order to facilitate this, the propulsion system performance may instead be represented by a Net Propulsive Force (NPF). This term typically includes the force terms associated with the engine cowl and afterbody, spillage drag, and interference drag. NPF can be defined as in Equation 2, where $\phi_{\text {nacelle }}$ is the nacelle force, $F_{G 9}$ is the gross thrust, and $F_{G 1}$ is the force that acts on the inlet (Figure 1) [20].

$$
\mathrm{NPF}=F_{G 9}-F_{G 1}-\phi_{\text {nacelle }}
$$

For a boundary layer ingesting system, a portion of the airframe profile drag could be accounted for in the propulsion system drag. This portion of airframe drag is encompassed by the streamtube which enters the propulsion system inlet. It could be argued that the profile drag of the entire airframe section prior to the inlet must be accounted for as a component of the propulsion system drag. However, this complicates the calculation of the propulsion system performance during the various flight phases of the aircraft, as the separation between the airframe and propulsion system is unclear. Inclusion of the entire upstream flow would result in an engine performance calculation that required aircraft performance and operating data for every point in the flight of interest. At the opposite extreme, where no portion of the airframe is included in the propulsion system control volume, the aircraft drag calculation would be reliant on engine performance. At off-design conditions or at inlet mass flow ratios not equal to unity, the engine suction may accelerate or decelerate flow into the inlet. This would result in changes in the skin friction drag directly before the inlet as a function of the propulsion system performance, rather than aircraft performance. It is therefore clear that, in order to adequately split the aircraft and 
propulsion system thrust and drag accounting, a suitable interface point, $i$, should be chosen (Figure 1). This interface should approximately indicate the region where engine thrust and drag is no longer a function of the aircraft condition, and vice versa. This station has been suggested to lie in the order of approximately twice the inlet height ahead of the engine highlight plane [22, 23]. An equation to calculate the net propulsive force of the propulsion system may be created using this station definition:

$$
\mathrm{NPF}=F_{G 9}-F_{G i}-\tau_{w} S_{\mathrm{wet}}-D_{\text {nacelle }}
$$

Where $\tau_{w} S_{\text {wet }}$ represents the skin friction drag from the wetted surface area between stations $i$ and the inlet highlight (Station 1). As this portion of drag is now accounted for in the propulsion system, it must be separated from the calculated aircraft drag. The value of $\tau_{w} S_{\text {wet }}$ will account for only a small proportion of the total drag of the aircraft, unless the propulsion system becomes very large.

The net propulsive force formulation establishes an equation for performance assessment of a BLI propulsion system. The following subsections work through the process to obtain the parameters necessary for the final NPF result. This process begins with an estimation of the boundary layer characteristics at the interface point, $i$, followed by the characteristics for the inlet stream. These inlet stream characteristics are subsequently implemented in a conventional one dimensional gas dynamics method for calculation of the propulsion system NPF.
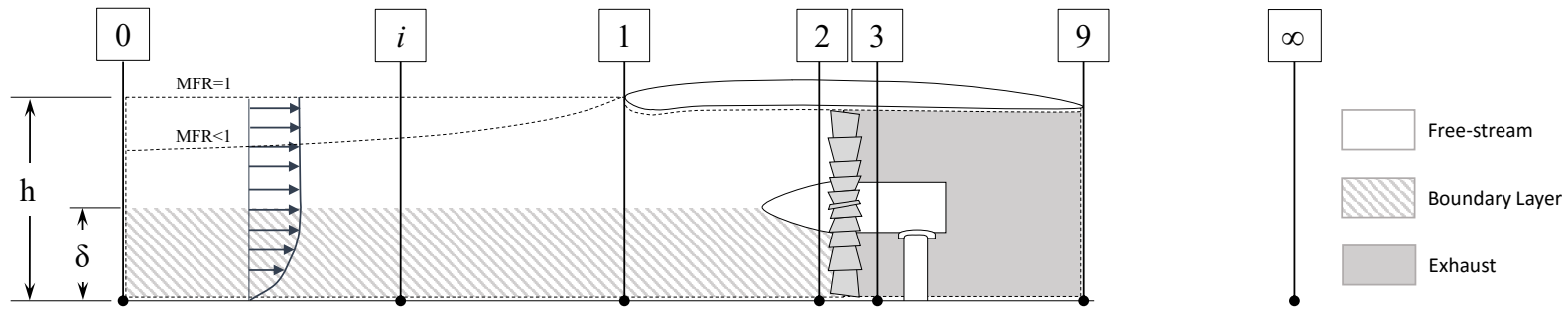

Figure 1: Propulsion system control volume and station definition.

\section{B. Boundary Layer Characteristics}

Equations which describe the flow in a boundary layer can be derived from the Navier-Stokes equations for viscous flow. These equations reduce to a more easily solvable form than the full Navier-Stokes equations through the application of appropriate limits to the integrals and with the use of velocity profile approximations [24]. The $1 / n^{\text {th }}$ power law relationship provides one form of velocity profile approximation, where a typical value used to approximate a fully developed boundary layer is $n=7$ [25]. As one potential alternative, Pohlhausen's approximation of the boundary layer velocity profile applies a fourth order polynomial to represent the boundary layer velocity profile and makes use of the pressure gradient in the external flow [26]. A number of methods are also available for estimation of the boundary layer thickness, $\delta$, and characteristics. One such boundary layer thickness estimation is a simple turbulent flat plate approximation of the boundary layer (Equation 4) [27]. The flow regime for the case under consideration determines whether laminar or turbulent boundary thickness assumptions should be applied. Alternative functions may provide more suitable estimations of the boundary layer thickness for the specific configuration and flow regime at which the system operates.

$$
\delta=\frac{0.37 x}{R e_{x}^{1 / 5}}
$$

A number of additional dimensions that define the boundary layer are available: the displacement thickness $\left(\delta^{*}\right)$, momentum thickness $(\theta)$, and energy thickness $\left(\theta^{*}\right)[28]$ :

$$
\begin{gathered}
\frac{\delta^{*}}{\delta}=\int_{0}^{\infty}\left[1-\frac{\rho_{y}}{\rho_{0}} \frac{u_{y}}{u_{0}}\right] \mathrm{d} y \\
\frac{\theta}{\delta}=\int_{0}^{\infty} \frac{\rho_{y}}{\rho_{0}} \frac{u_{y}}{u_{0}}\left[1-\frac{u_{y}}{u_{0}}\right] \mathrm{d} y \\
\frac{\theta^{*}}{\delta}=\int_{0}^{\infty} \frac{\rho_{y}}{\rho_{0}} \frac{u_{y}}{u_{0}}\left[1-\left(\frac{u_{y}}{u_{0}}\right)^{2}\right] \mathrm{d} y
\end{gathered}
$$


Each boundary layer thickness term represents the distance by which the surface would have to be displaced in an inviscid flow in order to result in the same mass flow, momentum or kinetic energy as the viscous flow. Given the definition of these terms, the boundary layer flow characteristics may be represented as non-dimensional parameters [23]:

$$
\begin{gathered}
\mathrm{MAG}=\frac{\dot{m}_{\mathrm{BL}}}{\rho_{0} u_{0 i} \delta w}=\int_{0}^{1} \frac{\rho_{y}}{\rho_{0}} \frac{u_{y}}{u_{0}} \mathrm{~d}(y / \delta)=1-\frac{\delta^{*}}{\delta} \\
\mathrm{MOG}=\frac{\mathrm{mom}_{\mathrm{BL}}}{\rho_{0} u_{0 i}^{2} \delta w}=\int_{0}^{1} \frac{\rho_{y}}{\rho_{0}}\left(\frac{u_{y}}{u_{0}}\right)^{2} \mathrm{~d}(y / \delta)=1-\frac{\delta^{*}}{\delta}-\frac{\theta}{\delta} \\
\mathrm{KEG}=\frac{\mathrm{KE}_{\mathrm{BL}}}{0.5 \rho_{0} u_{0 i}^{3} \delta w}=\int_{0}^{1} \frac{\rho_{y}}{\rho_{0}}\left(\frac{u_{y}}{u_{0}}\right)^{3} \mathrm{~d}(y / \delta)=1-\frac{\delta^{*}}{\delta}-\frac{\theta^{*}}{\delta}
\end{gathered}
$$

The non-dimensional groups represented in Equations 8-10 are referred to as the mass flow group (MAG), the momentum group (MOG), and the kinetic energy group (KEG). The groups define the flow characteristics in a unit area of the boundary layer flow relative to the same unit area in a free-stream flow. Each of the boundary layer characteristics, $\dot{m}_{\mathrm{BL}}, \mathrm{mom}_{\mathrm{BL}}$ and $\mathrm{KE} \mathrm{BL}_{\mathrm{L}}$, is subject to a deficit relative to the equivalent free-stream flow characteristics. In addition to deficits in the mass flow, momentum and kinetic energy, flow in the boundary layer experiences a certain measure of total pressure deficit. This can be calculated as a mass-averaged value:

$$
\frac{\bar{P}_{B L}}{P_{0}}=\frac{1}{\dot{m}_{B L}} \int_{B L} \frac{P_{y}}{P_{0}} \mathrm{~d} \dot{m}
$$

The average velocity of flow through the boundary layer may be defined in a similar integral manner:

$$
\frac{\bar{u}_{B L}}{u_{0}}=\frac{1}{\dot{m}_{B L}} \int_{B L} \frac{u_{y}}{u_{0}} \mathrm{~d} \dot{m}
$$

The primary purpose of this step in the process is to output three relevant boundary layer characteristics: the mass flow, average velocity, and the average total pressure relative to the free-stream total pressure. Any of the numerous methods available for determining the boundary layer characteristics may be applied, provided that they produce the required boundary layer characteristics.

\section{Inlet Properties for a BLI System}

Flow ingested by the propulsion system may be more or less than the entire boundary layer flow. Hence, an additional step is required in the process in order to establish these inlet flow characteristics. Assuming a rectangular streamtube with height $h$ and width $w$, there are three possible options that can be considered:

1. Ingest only the boundary layer $(h / \delta=1)$.

2. Ingest free-stream and the boundary layer $(h / \delta>1)$.

3. Ingest a portion of the boundary layer $(h / \delta<1)$.

In the first case, the characteristics of the ingested flow can be derived using the equations summarised in the previous subsection. In the case of an inlet which ingests only a portion of the boundary layer, the upper limit of the integrals of Equations $8-10$ becomes $h / \delta$, the ratio of streamtube height to boundary layer thickness. In the final case, ingested flow characteristics must take into account the combination of both free-stream and boundary layer flow. The boundary layer characteristics and profile can be calculated as usual. However, the total inlet mass flow of the inlet stream is the sum of both the free-stream (flow through the area $(h-\delta) w)$ and boundary layer mass flow:

$$
\frac{\dot{m}_{\text {total }}}{\rho_{0} u_{0 i} \delta w}=\frac{\dot{m}_{\mathrm{FS}}+\dot{m}_{\mathrm{BL}}}{\rho_{0} u_{0 i} \delta w}=\left(\frac{h}{\delta}-1\right)+\mathrm{MAG}
$$

Unlike mass flow, the total pressure deficit and average velocity of the flow are terms that are averaged over the entire inlet stream. Calculation of each of these flow characteristics follows on from the definitions established in Equations 11 and 12. Therefore, the combined total pressure deficit of the boundary layer and free-stream flow is a mass-averaged value integrated over the entire stream (Equation 14). However, total pressure for flow outside the boundary layer is constant $\left(P_{y}=P_{0}\right)$. This splits the integral into 
boundary layer ( $y$ between 0 and $\delta$ ) and free-stream flow sections ( $y$ between $\delta$ and $h$ ). The solution to the total pressure integral of the inlet flow may be represented in the non-dimensional form of Equation 16.

$$
\begin{gathered}
\frac{\bar{P}_{i}}{P_{0}}=\frac{1}{\dot{m}_{B L}} \int_{0}^{h} \frac{P_{y}}{P_{0}} \mathrm{~d} \dot{m} \\
\frac{\bar{P}_{i}}{P_{0}}=\frac{1}{\dot{m}_{\text {total }}}\left[\int_{0}^{\delta} \frac{P_{y}}{P_{0}} \mathrm{~d} \dot{m}+\int_{\delta}^{h} \mathrm{~d} \dot{m}\right] \\
\frac{\bar{P}_{i}}{P_{0}}=\frac{\left(\frac{h}{\delta}-1\right)+\mathrm{MAG}\left(\frac{\bar{P}_{B L}}{P_{0}}\right)}{\left(\frac{h}{\delta}-1\right)+\mathrm{MAG}}
\end{gathered}
$$

The average velocity in the combined stream may be likewise calculated by integrating over the entire inlet stream (Equation 17). Similar to the total pressure deficit, the velocity outside of the boundary layer in the local free-stream flow is constant $\left(u_{y}=u_{0}\right)$, which splits the integral into free-stream and boundary layer flow components.

$$
\frac{\bar{u}_{i}}{u_{0}}=\frac{1}{\dot{m}_{B L}} \int_{0}^{h} \frac{u_{y}}{u_{0}} \mathrm{~d} \dot{m}
$$

Each of the three defined terms, $\dot{m}_{\text {total }}, \bar{u}_{i}$, and $\bar{P}_{i} / P_{0}$, is a key component of the net propulsive force calculation through the definition of $F_{G}$ (Equation 1). The representation of each of the terms as averaged and total values for the stream enables the integration of the boundary layer characteristics within conventional methods for propulsion system performance.

\section{Ingested Drag of a BLI System}

A key factor in the efficiency benefit of a BLI system is the reduction in airframe drag due to the reenergisation of the wake. The drag of the airframe may be split into two components: the ingested drag, $D_{\text {ingested, }}$ and the uningested drag of the airframe, $D_{\text {aircraft }}$. The clean aircraft drag may therefore be represented as the sum of the ingested and uningested drag components (Equation 18). In steady level flight the propulsion system is required to produce only enough net thrust to counteract the drag not recovered by wake re-energisation, $D_{\text {aircraft }}$, rather than the entire drag of the clean aircraft. However, the net propulsive force is required to counteract the entire system drag. This definition highlights that the thrust $\left(F_{N}\right)$ requirement of a BLI system is a value dependant on its configuration and location, despite a constant NPF.

$$
D_{\text {aircraft,clean }}=N P F=D_{\text {ingested }}+D_{\text {aircraft }}
$$

Plas [22] references a method for calculation of the drag recovered by wake re-energisation based on a modified von Kármán momentum equation (Equation 19). The calculation of ingested drag makes use of boundary layer characteristics at the interface point and the average boundary layer shape factor, $H$ (Equation 20), between the interface point (Station $i$ ) and the wake (Station $\infty$ ):

$$
\begin{gathered}
D_{\text {ingested }}=\rho_{B L} u_{B L}^{2} \theta w\left(\frac{\bar{u}_{\mathrm{BL}}}{u_{0}}\right)^{H_{\text {avg }}} \\
H_{\text {avg }}=\frac{H_{i}+1}{2}=\frac{1}{2}\left(\frac{\delta^{*}}{\theta}+1\right)
\end{gathered}
$$

To maintain consistency with the boundary layer characteristic definitions used here, the ingested drag is defined non-dimensionally through substitution for MOG (Equation 9):

$$
\frac{D_{\text {ingested }}}{\rho_{0} u_{0 i}^{2} \delta w}=\mathrm{MOG} \cdot \frac{\theta}{\delta}\left(\frac{\bar{u}_{\mathrm{BL}}}{u_{0}}\right)^{H_{a v g}}
$$

Assuming that the airframe drag remains constant, drag ingested (or recovered) by the propulsion system reduces the required thrust output in comparison to an equivalent propulsion system in free-stream flow. At first glance, an engine which ingests only free-stream flow can therefore appear a better option than a BLI engine, as $F_{N}$ is greater. However, accounting for the ingested drag on the engine side of the NPF equation balances out this difference. In steady, level flight this means that the propulsion system NPF should be equal to the drag of the clean aircraft, $D_{\text {aircraft,clean, the drag of the airframe excluding }}$ 
the propulsion system (Equation 22). The BLI system is therefore sized to achieve the NPF required by the aircraft design. Using this definition of NPF, both a propulsion system in free-stream flow and a BLI propulsion system would be required to produce the same net propulsive force on the same aircraft configuration.

$$
\mathrm{NPF}=F_{G 9}-F_{G i}-\tau_{w} S_{\text {wet }}-D_{\text {nacelle }}+D_{\text {ingested }}=D_{\text {aircraft,clean }}
$$

The calculation of each term in the net propulsive force equation requires the application of the previously described methods for estimation of the characteristics of a boundary layer. These boundary layer characteristics are used to obtain the inlet characteristics of the system, in addition to ingested drag. Unlike a system operating in free-stream flow, the inlet stream characteristics are a function of the inlet stream size through the $h / \delta$ term.

\section{E. Representing the Efficiency of a BLI System}

Measures of efficiency are a necessary component of the assessment of a propulsion system. For a BLI system in particular, the performance relative to a conventional propulsion system must be improved by a sufficient margin to justify the adoption of the technology. However, appropriate representations of efficiency are required to define the efficiency of a BLI system. Propulsive efficiency $\left(\eta_{\text {propulsive }}\right)$ contributes to the overall efficiency parameter of a conventional propulsion system. In the standard form used in propulsion system performance, propulsive efficiency quantifies the useful propulsive power output as a percentage of the power available from the free-stream flow. For a conventional propulsion system, this can be calculated using the following formula:

$$
\eta_{\text {propulsive }}=\frac{2 u_{0}}{u_{0}+u_{j}}
$$

The formula highlights one of the drivers of propulsion system design: maximising the propulsive efficiency necessitates minimising the exhaust jet velocity. However, this is associated with a reduction in the specific thrust of the propulsion system. In the case of a BLI propulsion system there are three velocities that define the propulsive efficiency, as the flow entering the propulsion system is not equal to the flow available in the free-stream. These velocities are the velocity of the air entering the inlet, $\bar{u}_{i}$, the velocity of the exhaust jet, $u_{j}$, and the velocity of the free-stream flow, $u_{0}$. The propulsive efficiency of a boundary layer ingesting system therefore cannot be defined in the same manner as that of a free-stream engine. As the useful propulsive power is in terms of the local inlet stream, the propulsive efficiency of the system is defined relative to these terms [29]:

$$
\eta_{\text {propulsive }, \mathrm{BL}}=\frac{2 u_{0}}{\bar{u}_{i}+u_{j}}
$$

In the case of a purely wake filling BLI propulsor, the exhaust velocity is equal to the free-stream velocity $\left(u_{j}=u_{0}\right)$. However, unlike a conventional propulsion system, the inlet velocity is less than the free-stream $\left(\bar{u}_{i}<u_{0}\right)$. The denominator of the propulsive efficiency equation is therefore less than the numerator, as $\bar{u}_{i}+u_{j}$ is equal to $\bar{u}_{i}+u_{0}$, which is less than $2 u_{0}$. In this configuration the propulsive efficiency as defined in Equation 24 is greater than 100\%. An increase in the momentum deficit leads to further reductions in $\bar{u}_{i}$ which results in an increase in propulsive efficiency. Even in a BLI system with a higher exhaust velocity, the propulsive efficiency will be greater than that of an equivalent propulsion system in free-stream flow. This is due to the discrepancy between $u_{0}$ and $\bar{u}\left(\bar{u}_{i}<u_{0}\right)$ and the fact that, for the same specific thrust, $u_{j}$ for a BLI propulsion system is lower than that of a propulsion system in free-stream flow. $\bar{u}_{i}$ tends to $u_{0}$ as the momentum deficit reduces, and the propulsive efficiency returns to the conventional definition.

Propulsive efficiency is not the only descriptor of performance and efficiency. A high propulsive efficiency in a BLI system may result from a thick boundary layer with a high momentum deficit. However, this may be matched by distortion related inefficiencies elsewhere which are detrimental to the overall system performance. The performance benefit of a BLI system can instead be represented in terms of a power saving coefficient, as proposed by Smith (Equation 25) [4]. This enables the assessment of the benefits of the propulsion system as a whole relative to a comparable system in free-stream flow.

$$
\mathrm{PSC}=\frac{P_{\text {ref }}-P_{\mathrm{BLI}}}{P_{\text {ref }}}
$$

Where $P_{\text {ref }}$ indicates the power consumption of a podded propulsion system operating in freestream flow required to propel an aircraft with drag $D_{\text {aircraft,clean. }} P_{\mathrm{BLI}}$ indicates the power consumption of a BLI 
propulsion system required to propel the same aircraft. A positive power saving coefficient represents a BLI system with a power consumption that is lower than that of a free-stream propulsion system. The power saving coefficient representation of efficiency encompasses any losses within the system, and is able to represent the changes of a BLI system relative to an equivalent free-stream option. Smith found that in some cases that the PSC for propulsion system could be in the region of $7 \%$, for the configurations considered [4]. Plas notes that the power saving coefficient will reduce as system loss is increased and can negate the benefit of a BLI system. Nonetheless, the configuration considered had the potential for a power saving of up to approximately $10 \%$ when $25 \%$ of the airframe drag was ingested [5]. The PSC parameter has been used to assess the impact that configuration changes or flow distortion have on the system as a whole. A system that results in a PSC less than zero may be concluded to be non-viable, as a free-stream propulsion system would be a more efficient option.

\section{F. Propulsion System Sizing and Design Point Performance}

The design point simulation of a boundary layer ingesting propulsion system follows the method progression shown in Figure 2. The first step in the process establishes the local flow characteristics (including Reynolds number, boundary layer thickness and local free-stream velocity at the edge of the boundary layer). These local conditions provide support for assessment of how the spanwise flow variation influences performance. Subsequently the boundary layer characteristics and inlet stream characteristics may be determined. Finally, the inlet stream characteristics are used to calculate the performance of the propulsor using conventional one dimensional gas dynamics. Inlet properties are defined as average and total inlet stream values through the calculations described in the previous sections. The specific configuration under assessment is required in order to define the local properties required for inlet flow calculations. Two key representations of the location effects have been identified that influence local flow characteristics. The first of these is a variation in local velocity $\left(u_{0 i}=f(x, y)\right)$. As an example configuration, in a blended wing body type aircraft the aerofoil cross-section results in a velocity distribution over the airframe induced by the pressure distribution over the aircraft surface [30]. Flow over an aerofoil accelerates from the leading edge up to a point along the aerofoil surface, followed by a subsequent deceleration. Local velocity at the edge of the boundary layer, $u_{0 i}$, is therefore strongly dependent on the airframe configuration. The second location effect that was considered is the change in boundary layer thickness. The boundary layer thickness is a function of both the Reynolds number and the surface length before the inlet (Equation 4). Inlets mounted at the leading edge of an airframe will therefore ingest little to no boundary layer, whilst inlets mounted at the trailing edge (or in the wake) will have a noticeable thicker boundary layer available.

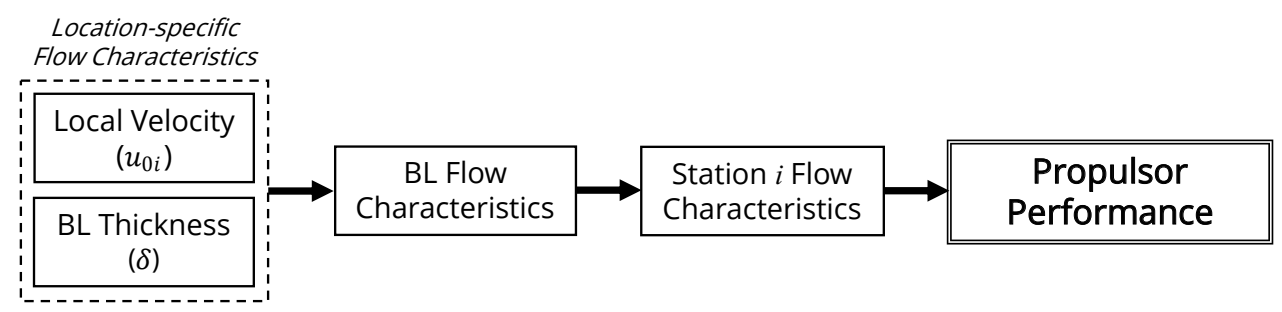

Figure 2: BLI propulsor simulation method.

Nacelle design is an additional important factor for the integrated engine performance and the NPF model. For the purposes of this research a simple nacelle design was implemented for nacelle drag estimation. This included a NACA-1 forebody, a straight midbody, and a circular arc afterbody. As will be demonstrated later in this research, the nacelle drag results in an approximately step increase in the power consumption of the BLI system. Alternative nacelle configurations will change the magnitude of this increase, with the potential for designs that minimise the drag of a BLI nacelle. Due to the integrated configuration, the lower surface of a BLI system is embedded in the airframe. The wetted surface area and hence nacelle drag of a BLI propulsion system may therefore be lower than that of a conventional podded engine.

The boundary layer theory discussed in the previous section represents the impact of the boundary layer on inlet stream characteristics as three changes relative to a free-stream propulsion system: a total pressure deficit, a reduction in the inlet velocity, and a reduction in mass flow. Unlike 2- or 3-D methods, additional inlet pressure loss due to the boundary layer and compressor efficiency loss due to distortion are not directly represented. These may instead be introduced into performance calculations as averaged 
numerical approximations.

Similar to a conventional propulsion system, the size of a propulsor is determined by the propulsive force required. However, in the case of a BLI system, changes to the propulsor and inlet dimensions will influence the averaged flow characteristics at the interface point. Estimation of the size of a BLI propulsor using the method developed in this research therefore necessitates a procedure that solves for a propulsor size which produces the required net propulsive force.

\section{Case Study on the N3-X}

A case-study was performed on the N3-X aircraft to demonstrate the application of the model to an aircraft configuration. The N3-X (Figure 3) is a blended/hybrid wing body aircraft developed by NASA propelled by a boundary layer ingesting distributed propulsor array. The array is powered by a pair of turbogenerators via a superconducting electrical system [10]. The distributed propulsor array produces the majority of the aircraft thrust, with the turbogenerators producing enough thrust to counter their drag. The propulsion system was designed for a Mach number of 0.84 at an altitude of 30,000 ft. Previous simulations of the N3-X relied on an uninstalled analysis of the propulsion system and a net thrust perspective $[10,13,31]$. The case study attempted to address a number of limitations with previous simulations of the aircraft propulsion system:

- Direct representation of the airframe drag recovered by wake re-energisation

- Spanwise variation in flow characteristics along the length of the array

- Reduced reliance on CFD data for performance calculations

The combination of these factors supports the development of a propulsion system model that may be integrated within an aircraft performance model. In addition, it enables the assessment of multiple aircraft and propulsion system configurations.

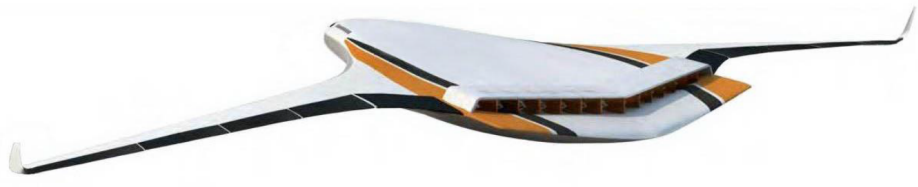

Figure 3: NASA's N3-X Aircraft [32].

The propulsor array has an inlet set at $85 \%$ of the centreline chord and parallel to the trailing edge [10]. A combination of the highly swept fuselage leading edge and the angled nature of the propulsor array results in a significant change in chord length and local Mach number between the centreline propulsor and the propulsors at the extreme edges of the array. Due to the nature of the aircraft shape and airflow, each propulsor inlet experiences different boundary layer and air flow characteristics at the interface point (Figure 4). The inclusion of these span-related effects means that each fan in the propulsor array performs differently dependent on the location and configuration. These differences introduce an additional level of complexity into the simulation of the propulsor array. The complexity can be simplified with the assumption that conditions are mirrored about the centreline. Nonetheless, this does imply that each propulsor must be individually designed if optimisation of the propulsion system performance is required. The rapid assessments possible using the developed method enable multiple configurations to be assessed in order to identify where the optimum may lie.

The case study made use of publicly available data and research on the aircraft: the aircraft and propulsor array configuration [10], and the boundary layer profiles at the centreline of the airframe [33]. The aircraft configuration provided the reference lengths ( $x$, distance from the aircraft leading edge) necessary for estimation of the boundary layer thickness. As an initial estimate, the turbulent flat plate assumption was applied (Equation 4). To account for the discrepancy between the flat plate assumption and the actual aircraft configuration, the boundary layer thickness was scaled by a constant to match the available boundary layer profiles. The boundary layer profiles also provided an estimation of the local free-stream velocity at the edge of the boundary layer on the aircraft centreline. The velocity profile provided the local free-stream Mach number at the edge of the boundary layer from $x / c=0.6$ to $x / c=1.0$, where $c$ is the centreline chord length. In the absence of a full CFD data analysis of the airframe, the velocity profile was extended to encompass the entire airframe. This included the assumption that the local free-stream velocity at the edge of the boundary layer would be equal at any axial distance $x_{0}$ from the aircraft nose. A more detailed estimate of airframe boundary layer and 
velocity distribution may be calculated using a more complete analysis of the airframe. However, the publicly available data was applied to demonstrate the analysis possible using the limited information that might be available at an early design stage. This is in keeping with the intended application of the method as a preliminary design tool.
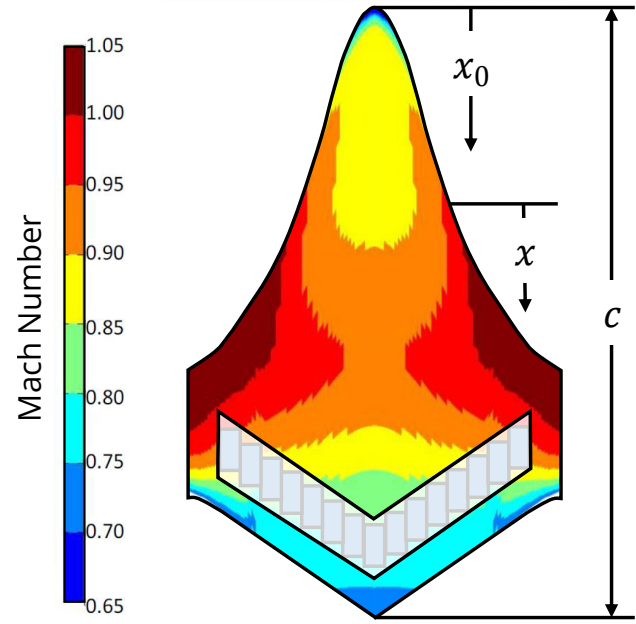

(a) Mach number profile [33].
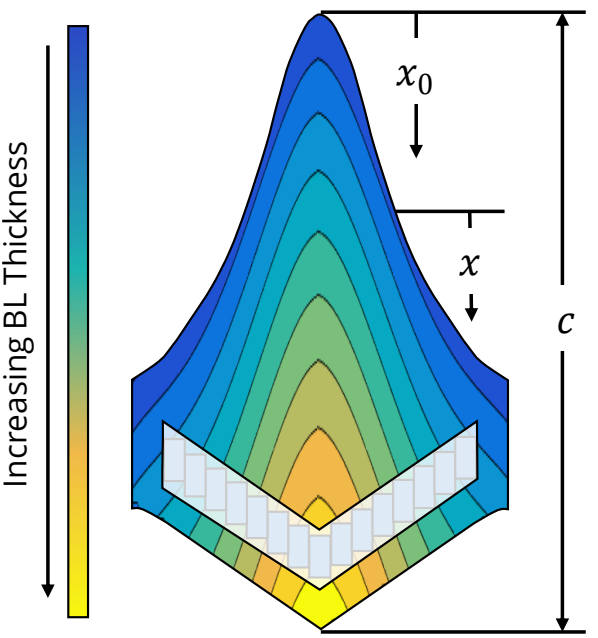

(b) Fuselage boundary layer thickness.

Figure 4: N3-X airframe Mach number profile and BL thickness.

\section{Model Validation}

In order to assess the applicability of the model, a validation procedure was performed. The N3$\mathrm{X}$ propulsor array has previously been simulated using a control volume method by Felder et al., the results of which were used to perform a preliminary validation [34]. The reference N3-X simulation did not include location-related or integration parameters. Therefore, in order to allow a like-for-like analysis, the propulsor performance was calculated assuming operation of an array of net thrust-producing (rather than NPF) propulsors at the centreline of the airframe.

A primary component of the developed method was the estimation of local properties using a boundary layer theory-based method, as opposed to a more complex CFD analysis. Therefore, the validity and accuracy of the predicted inlet stream characteristics was assessed. Reference [34] provides the average Mach number and total pressure deficit relative to free-stream for inlet streams of various heights, obtained from CFD data. An equivalent procedure to estimate flow characteristics was performed with the method of Section II over the range of streamtube heights provided by the reference. The results of this validation are shown in Figure 5. Comparison of the inlet stream properties to the NASA results shows an average difference in Mach number of 0.015 and an average $0.2 \%$ difference in boundary layer total pressure loss. This demonstrates that the inlet stream properties predicted by the analytical method match up closely with those predicted by the CFD derived results.

Subsequently, the overall performance output $\left(F_{N}\right)$ was compared to a sizing procedure performed in the reference [34]. The simulations performed in the reference attempted to predict the size of a BLI propulsor with a fixed thrust requirement. The reference method used a net thrust control volume combined with inlet flow characteristics derived from the CFD output in Figure 5 [13]. As has been noted, changing the size of the propulsors modifies the average inlet flow characteristics. Therefore, a procedure was required to solve for the inlet stream height $h$ which produces the required thrust, given the propulsor design parameters. In order to perform the comparison, a number of parameters were set to equal those reported in the reference for each simulation point: Total array thrust, fan pressure ratio, number of fans in the array (from which the net thrust of each propulsor may be calculated), inlet aspect ratio, and fan efficiency. The inlet height predicted by the model was found to match the trend shown by the NASA predictions with an average difference in the height of the propulsion system inlet stream of $3.8 \%$ (Figure 6 ). Both the reference method and the developed method apply a force control volume for performance calculation. Therefore, the validation demonstrated that the discrepancy in estimated inlet characteristics has only a minor impact on performance calculations. 


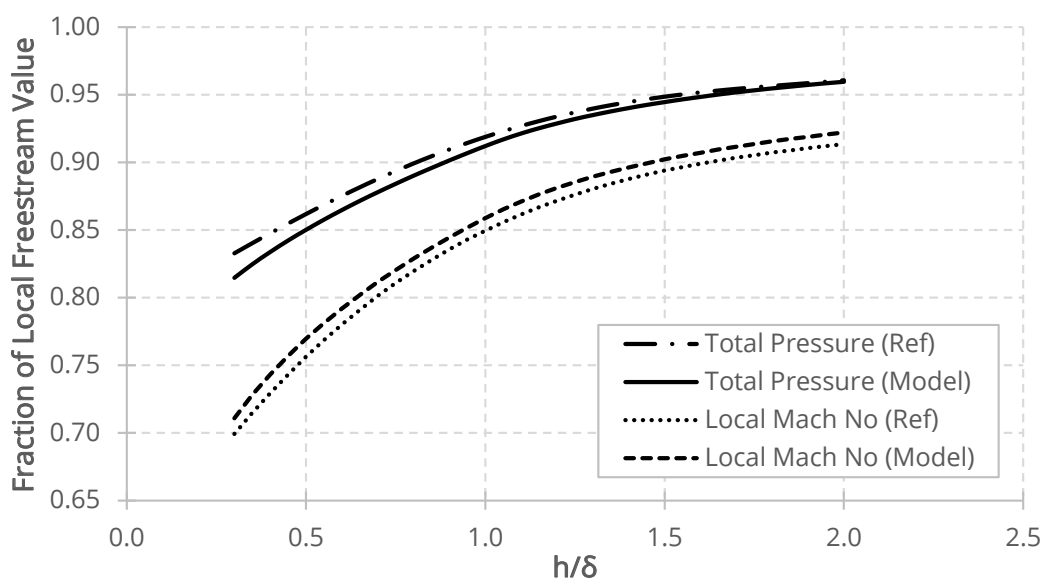

Figure 5: Inlet stream properties.

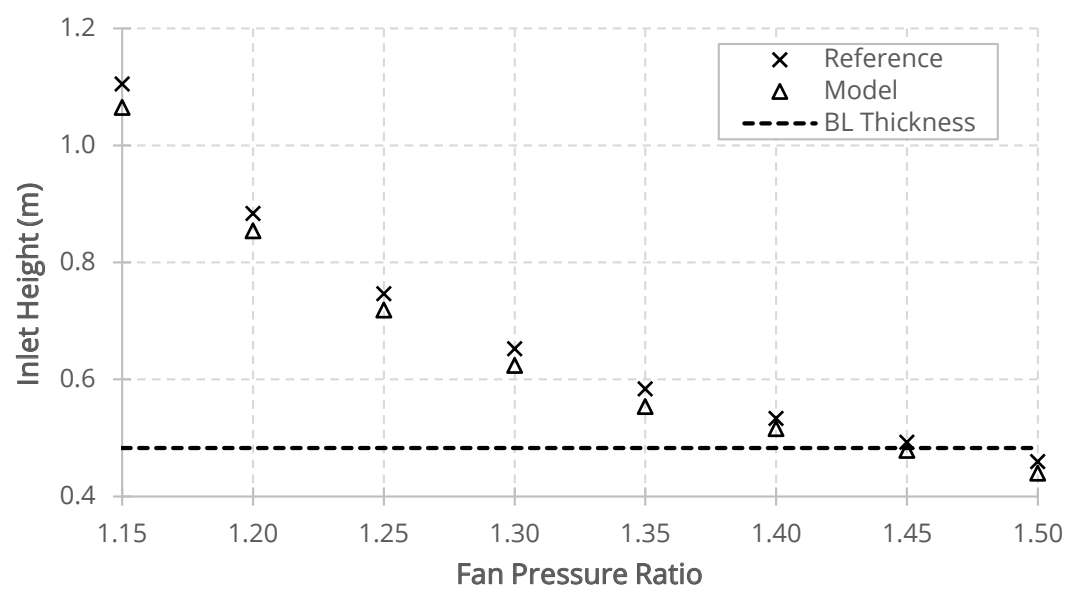

Figure 6: Inlet Height. 


\section{BLI Propulsor Design and Performance Trend Analysis}

The thrust produced by a propulsor in the array can be influenced in a number of ways. Assuming the same operating conditions and propulsor efficiency, an increase in propulsor size will increase the mass flow ingested by the propulsion system and therefore increase the total thrust. However, inlet properties are a function of the ratio of inlet height to boundary layer thickness. Hence, an increase in mass flow by an increase in inlet height will tend the performance towards that of a free-stream propulsion system (assuming a constant boundary layer thickness). Alternatively, the propulsor thrust can be increased with an increase in the fan pressure ratio. These two options are demonstrated in Figure 7 . Note that as the results of this subsection are in terms of net thrust, the nacelle drag and installation related factors of the NPF equations are neglected. This allows the performance of a configuration to be predicted before including installation-specific aspects.

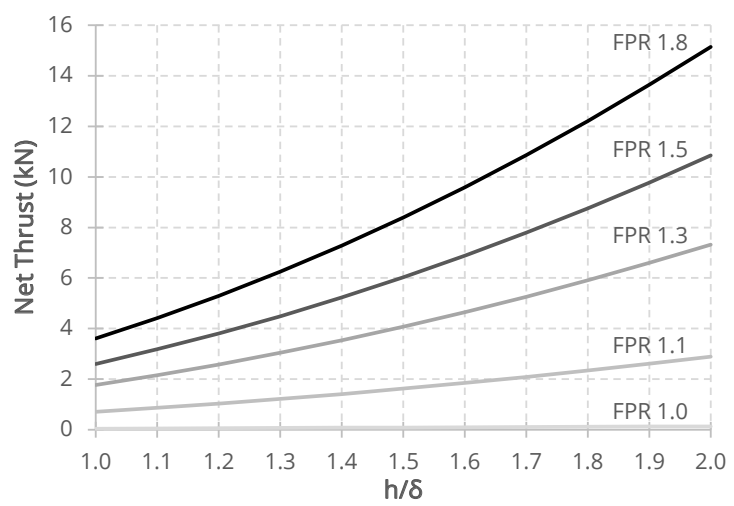

(a) Net Thrust.

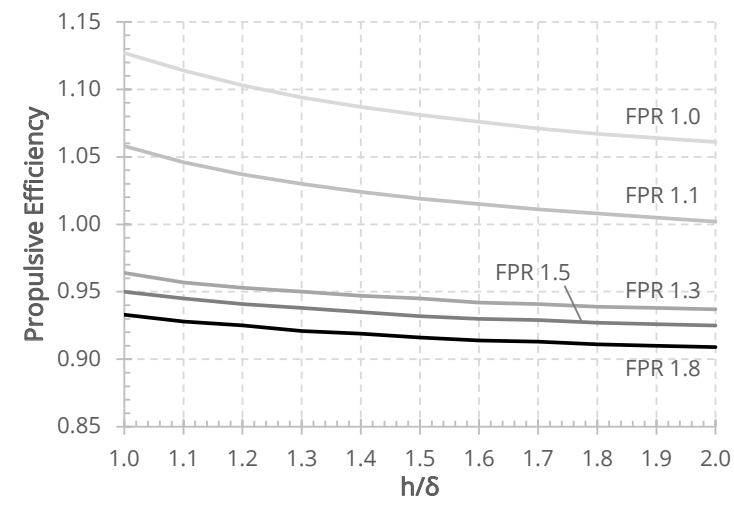

(b) Propulsive Efficiency.

Figure 7: Design charts for a propulsor at M0.84 and 30,000 ft.

A higher fan pressure ratio increases the exhaust velocity of the propulsion system. This results in an increase in specific thrust with a corresponding decrease in propulsive efficiency. The propulsive efficiency for the BLI system nonetheless remains high, and is greater than $100 \%$ for the simulated pressure ratios of 1.0 and 1.1. An increase in the inlet height increases the average velocity $\bar{u}_{i}$ at the inlet until it approaches the free-stream velocity $u_{0}$. This results in a drop in propulsive efficiency asymptotically approaching the point where $\bar{u}_{i}$ is equal to $u_{0}$ (Equation 17) and the propulsive efficiency is equal to that of a propulsion system in free-stream flow.

The design charts demonstrate one of the challenges in designing a thrust-producing BLI propulsor. Thrust increase obtained by simply increasing the mass flow (via scaling of the propulsor) results in a reduction in boundary layer benefit. A similar conclusion can be made from results shown by Liu et al [15], which demonstrates an increase in power saving coefficient as the BLI ratio (ratio of boundary layer mass flow to total mass flow, and related to the inverse of $h /$ delta) is decreased. Conventional propulsion system performance also dictates that a higher exhaust jet velocity through a higher fan pressure ratio will reduce system efficiency. A balance is therefore required between the two options.

\section{N3-X Case-Study Results}

The following section focuses on results obtained for the specific case of the N3-X. The net propulsive force requirement of the configuration is defined by the drag of the airframe, assuming a design point in steady level flight. The propulsor array is required to produce a net thrust of $85.82 \mathrm{kN}$ from 15 fans (5.72 $\mathrm{kN}$ net thrust per propulsor) [33], unless otherwise stated. The thrust requirement was defined based on the assumption that the net thrust required from the array is equal to the drag of the aircraft, scaled by a drag reduction factor of $7 \%$. This drag reduction factor accounts for the drag recovered by the wake re-energisation of the BLI system [10]. The original thrust requirement assumes that there is very little installation drag and that the drag of the nacelle is equal to the drag of the wetted surface area that would otherwise be exposed to free-stream air [33]. The required net thrust value was used to obtain the clean aircraft drag, equal to the NPF of Equation 22, as $92.28 \mathrm{kN}$. The design assessed in the following section will assume each propulsor produces the same NPF. 


\section{A. Boundary layer-related Losses}

The inherently distorted flow of the boundary layer will have a negative impact on propulsor performance, as additional inefficiencies and losses are introduced that would not be present with ingestion of only freestream flow. As the 1-D control volume method used here does not directly represent these additional losses, the sensitivity of performance to two such factors will be demonstrated. The first of these is a loss in total pressure through the inlet. This term accounts for the turbulent nature of the boundary layer, which may result in reductions in inlet efficiency on top of losses that would normally occur in a free-stream inlet. The second loss is a drop in fan efficiency due to the flow distortion at the fan face. The inlet flow has a non-uniform velocity and pressure profile introduced by the ingested boundary layer. This non-uniform flow can negatively impact the fan performance, especially for a fan that is not distortion tolerant. In addition, the flow profile can push the fan closer to surge, which necessitates a more generous surge margin for the design [5].

Figure 8 demonstrates the influence that losses have on the efficiency of a single propulsor at the centreline of the aircraft. The general trend demonstrates a reduction in PSC as the fan pressure ratio increases, similar to the analysis performed by Liu et al. [15]. For the low loss systems, a relatively lower pressure ratio corresponds to greater power savings. However, as the inlet total pressure loss increases, the power requirement of the fans operating with a relatively lower pressure ratio sharply increases. In order to achieve the required thrust, the propulsion system requires an increasingly large mass flow to compensate for a reduction in effective fan pressure ratio brought about by the inlet pressure loss. This is obtained by an increase in inlet height, which increases the ratio $h / \delta$ and brings the inlet average properties closer to free-stream. A higher fan pressure ratio reduces the mass flow required by the propulsion system and hence decreases the propulsion system size. However, power consumption is increased, as it is a function of both mass flow and enthalpy change. Nonetheless, a higher pressure ratio is more beneficial to power consumption in cases of high inlet pressure loss. An optimum point becomes apparent as the inlet pressure loss increases, defining the ideal fan pressure ratio to obtain the minimum power consumption of the propulsor.

Decreasing the efficiency of the fan results in an increase in the enthalpy change across the fan. However, there is little change in the thrust produced and hence no compensation by a change in mass flow ratio is required. This results in only a step increase in the power requirement of the fan as fan efficiency is decreased (Figure 8).

Although research has shown that ingesting a larger portion of airframe drag is beneficial for the power saving coefficient, specific design factors - such as the fan pressure ratio - will also have an influence. Low pressure ratio propulsors can ingest a greater amount of drag due to their greater size, but will have a lower PSC in high loss cases than alternative configurations, due to a higher power requirement. There is a point beyond which the free-stream propulsor is more efficient than a BLI propulsor. However, if one assumes that inlet and fan efficiency losses can be kept low, the power consumption of a BLI propulsor is lower than that of a propulsor producing the same net thrust from free-stream flow. This reflects conclusions drawn from previous research, which demonstrate a positive PSC for low loss BLI systems $[5,35]$.

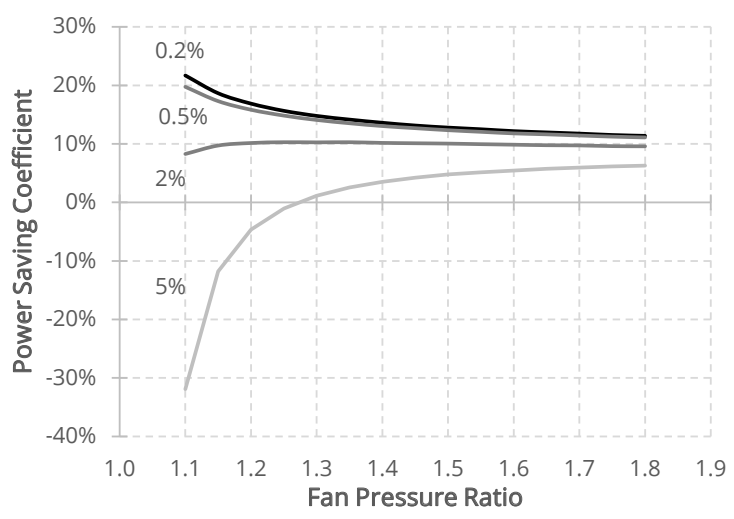

(a) Inlet pressure loss.

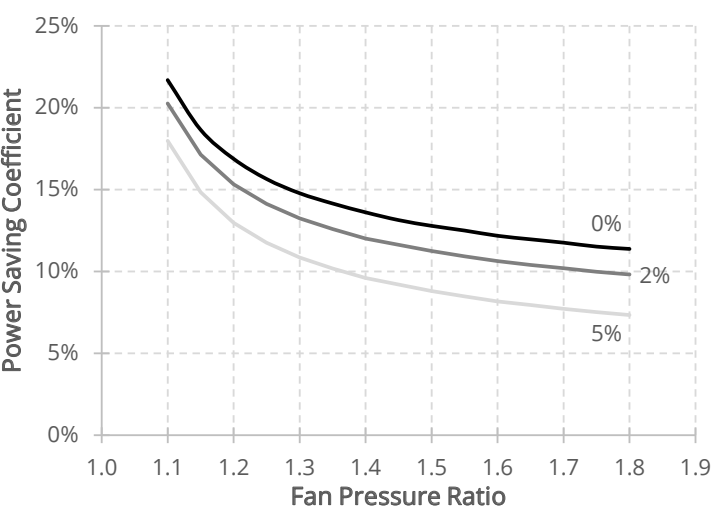

(b) Fan efficiency loss.

Figure 8: Influence of efficiency loss on PSC for a propulsor at M0.84 and 30,000 ft (neglecting nacelle drag). 


\section{B. Whole Array Performance}

The previous two sets of results have shown the performance of a single propulsor operating at the centreline of the aircraft. As one of the focuses was to assess the influence of property variation in the integrated system on performance, the following subsections will assess the propulsor array as a whole. In an ideal BLI case, each propulsor would ingest only boundary layer air $(h / \delta=1)$. In addition, the design would avoid extension into the high velocity and thin boundary layer flow that would occur at the outer edges fuselage section. In reality, the propulsors at the edges of the array see a significantly thinner boundary layer and a local velocity which approaches Mach 0.9. This has a negative impact on the efficiency of the propulsor and results in an increase the array power requirement and hence fuel burn of the propulsion system (Figure 9). The influence of the two previously defined locationrelated characteristics of the flow is broken down in Figure 9. Three fictitious cases were simulated to demonstrate how the span-related flow characteristics influence performance:

- No Span Effects - Each propulsor in the array ingests the same inlet flow conditions. This case is equivalent to each propulsor being located at the centreline of the airframe. Boundary layer thickness and local free-stream velocity at the edge of the boundary layer are constant. This hypothetical case represents the previous analyses performed on the N3-X propulsion system.

- $M_{i}$ Variation Only - Boundary layer thickness $\delta$ along the length of the array is equal to the centreline boundary layer thickness. The only spanwise variation in flow characteristics is due to the velocity profile over the airframe .

- $\delta$ Variation Only - Local Mach number at the edge of the boundary layer is equal to the centreline local Mach number. The only spanwise variation in flow characteristics is due to the change in boundary layer thickness as a result of the variation in fuselage length prior to the inlet.

The reduction of the boundary layer thickness along the array length results in a step increase in the power requirement of the array. This increase in power requirement results from the increase in $h / \delta$ along the array length, which tends the inlet flow characteristics to the characteristics of the free-stream flow. In addition, the thinner boundary layer reduces the wake re-energisation potential of the propulsion system. A higher value of $h / \delta$ therefore has a purely detrimental effect on PSC of the propulsion system. Higher local free-stream velocity results in an increase in momentum drag, $F_{G i}$. However the ingested drag is increased by the increasing local velocity (Equation 19). Assuming boundary layer properties remain constant, the higher local velocity results in a higher ingested drag. This is beneficial to performance despite an increase in $F_{G i}$. The combination of the two spanwise flow characteristics works to reduce the efficiency of the system as a whole.

Variation in fan pressure ratio establishes a clear optimum configuration for the array. The optimum is a result of a combination of the variation in flow characteristics along the array length and the power requirements of compression. An increase in fan pressure ratio reduces the mass flow necessary to produce the NPF required from the array. This shortens the array length and reduces $h / \delta$, through a reduction in the inlet area. However, the higher compression will increase the power consumption of the propulsion system. Despite the primarily beneficial effects that result from a shorter array, the reduced array length reduces the drag ingested by the propulsor array (Figure 9b), as the array extends over a smaller percentage of the airframe. The greatest proportion of airframe drag would be recovered by an array which stretched over the entire fuselage length. The maximum ingested drag corresponds with values predicted by Hardin et al, who suggest approximately $12 \%$ of the airframe drag could be recovered by an array on the N3-X upper fuselage [21].

The nacelle drag of the propulsion system is an additional factor in a full NPF assessment. The nacelle of an array such as that of the N3-X has a reduced wetted surface area in comparison to a conventional podded nacelle. This serves to reduce nacelle drag relative to a podded propulsion system. A PSC comparison is therefore no longer a like-for-like analysis. To account for this, the following results will be in terms of pure power consumption of the array.

Sizing the propulsion system with the full NPF equation increases the power consumption of the array, as each propulsor must compensate for the installation terms whilst still providing thrust (Figure 10). The addition of these installation terms further develops the 'hook' in the power requirement curve and shifts the optimum fan pressure ratio to a higher value. This optimum configuration is a result of the span-related flow characteristics identified in the previous analysis. Nacelle drag increases the net propulsive force required for the propulsion system, and hence increases the mass flow needed to produce the required force. The trade-off between array size and fan pressure ratio establishes a new configuration which minimises power consumption. Research suggests flow on the nacelle of the BWB may be prone to separation [36], therefore, the importance of a optimised nacelle design is further highlighted. 


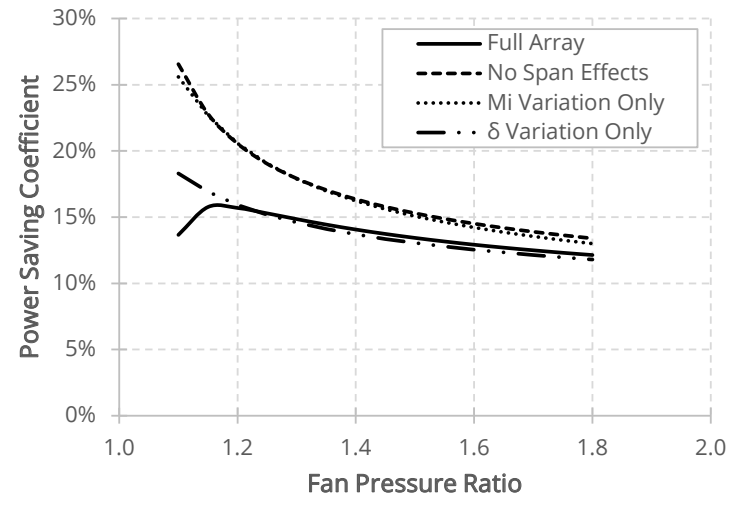

(a) Power saving coefficient.

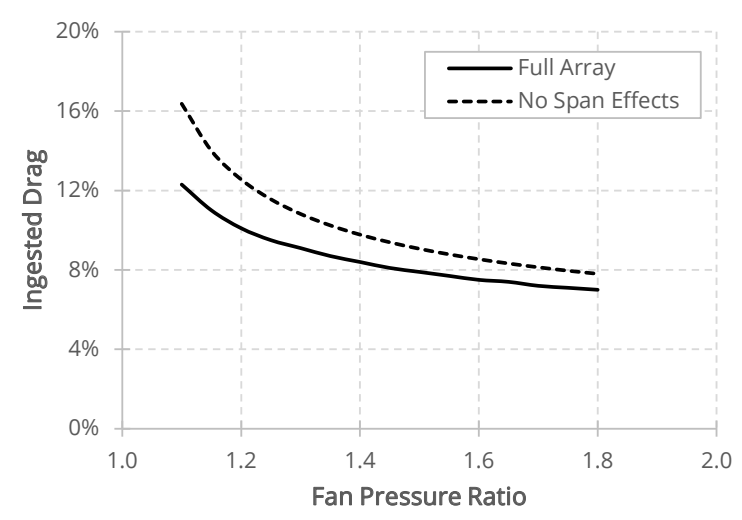

(b) Ingested drag.

Figure 9: Influence of span-wise flow characteristic variation on performance for an array with 15 fans at M0.84 and 30,000 ft (neglecting nacelle drag).

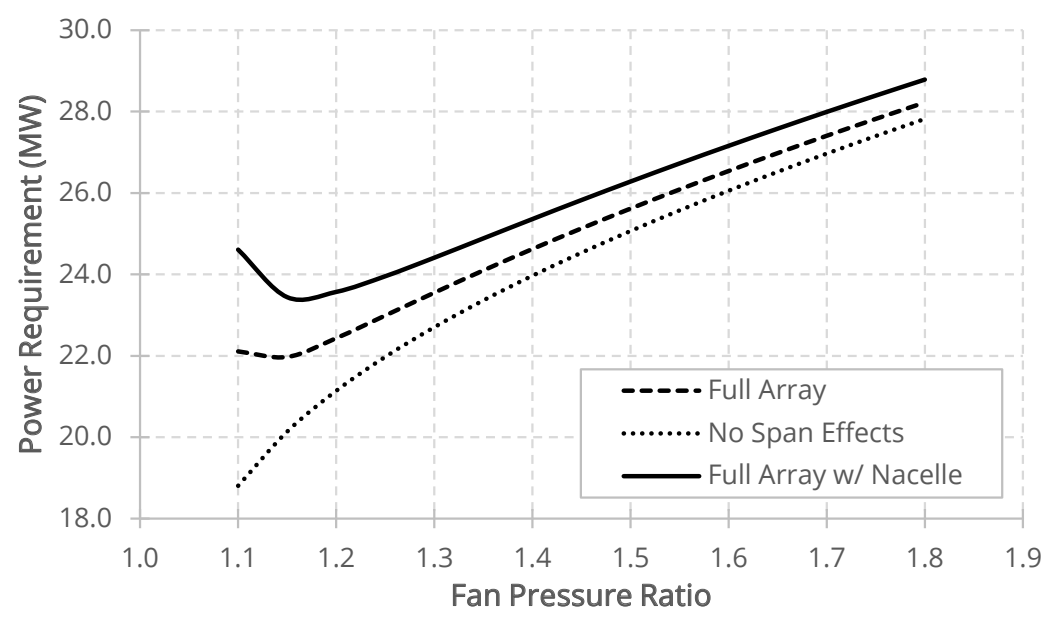

Figure 10: Power requirements for an array with 15 fans at M0.84 and 30,000 ft using full NPF equation. 


\section{Array Configuration}

Optimum configuration of the propulsion system has been shown to be dependant on the fan pressure ratio and thrust requirement of the design. However, the configuration also includes parameters such as the number of propulsors and the aspect ratio of each propulsor inlet. Tall, narrow inlets increases the ratio $h / \delta$, but will reduce array length, which avoids the flow on the outer edges of the fuselage. In contrast, short, wide inlets will bring the inlet height closer to the boundary layer height and ingest a relatively greater portion of the airframe drag. However, this will increase the array length. The trade-off between these costs and benefits determines the ideal configuration in terms of aspect ratio.

Given the link between the array configuration and performance, the optimum number of fans for the array is a key component of the ideal configuration. Assuming an equal fan pressure ratio for every propulsor in the array, any selected fan pressure ratio will correspond to a number of fans, $N_{\mathrm{fan}}$, which minimises the array power consumption (Figure 11). The $h / \delta$ parameter and array length, $L_{\text {array }}$, may be used to describe this outcome. An increase in $h / \delta$ tends the inlet characteristics towards the free-stream flow characteristics, which results in a reduction in the benefits of BLI. Therefore, for an individual propulsor with fixed fan pressure ratio, a reduction in $h / \delta$ is beneficial and reduces power consumption. If the inlet aspect ratio and the array NPF are to be maintained at a constant value, this may be achieved by an increase in the number of propulsor in the array. A greater number of propulsors reduces the average height of each propulsor, as the inlet area is spread over a greater array length. However, this extends the array into the relatively higher speed, thin boundary layer flow (high $h / \delta$ ) at the fuselage edge, which may result in a detriment to the overall power consumption of the array. A similar conclusion may be made with respect to the fan pressure selected for the array. A relatively higher fan pressure ratio propulsor has a smaller area (low $h / \delta$, short array), although the higher compression may result in an overall greater power consumption. A change in the inlet aspect ratio serves a similar purpose to changes in the $N_{\text {fan }}$ parameter, as aspect ratio will influence both the array length and $h_{\delta}$. A change in inlet aspect ratio establishes a new optimum for the array configuration (Figure 12). Each of the variables demonstrates that the optimum configuration is achieved where the results of changes in $h / \delta$ and array length are balanced.

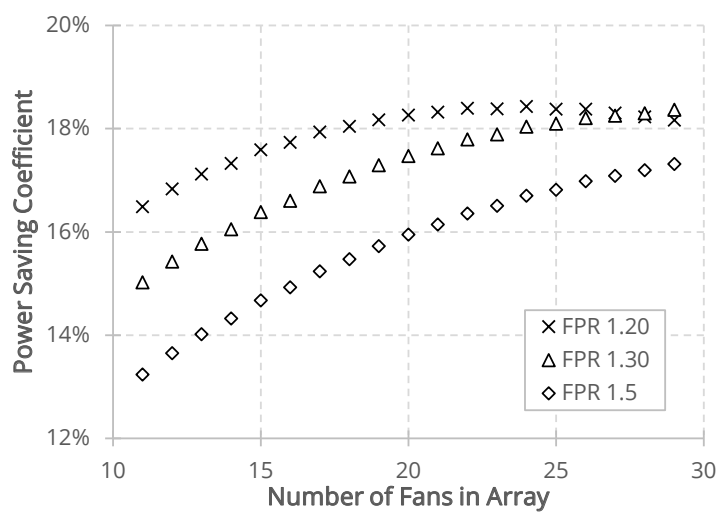

(a) Power Saving Coefficient.

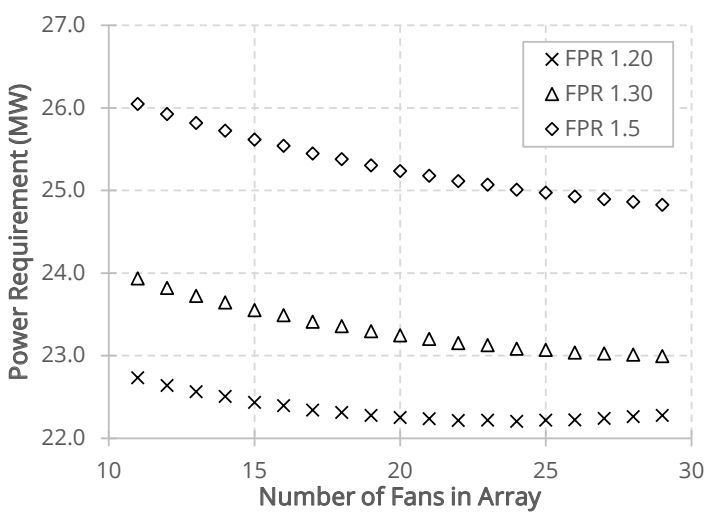

(b) Power Requirement.

Figure 11: Power for arrays with varying number of fans (neglecting nacelle drag).

\section{Conclusion}

This research has developed a method for the simulation of a boundary layer ingesting propulsion system, with particular emphasis on factors influenced by the integration of the system. Results have been presented for a distributed propulsion system operating on the N3-X, however, the method is non system-specific and may be adapted for use in alternative configurations.

A selection of simulation results have been presented which demonstrate how the boundary layer and span effects influence the performance of a propulsor array. The results display the importance of taking into account the span-wise variation in inlet characteristics along the length of the array. The addition of the flow characteristic variation results in an increase in the power required by the array, as the boundary layer thins and the entry Mach number increases along the length of the array. Despite a higher power requirement, the higher pressure ratio fans benefit from being less sensitive to inlet pressure losses. In 


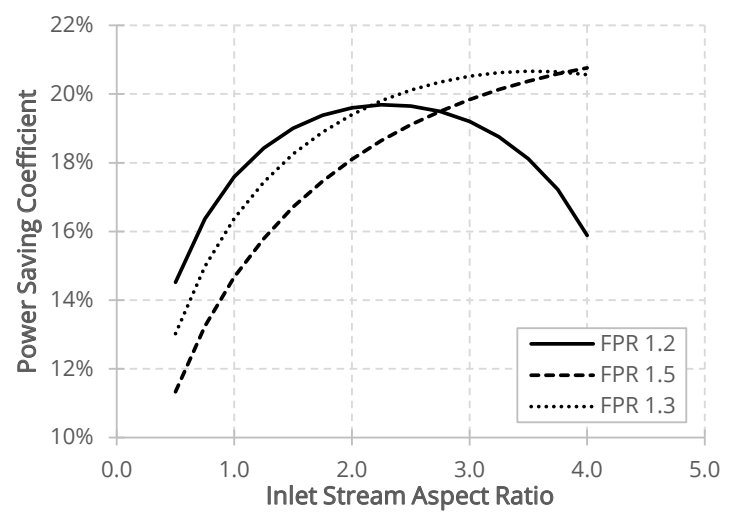

(a) Power Saving Coefficient.

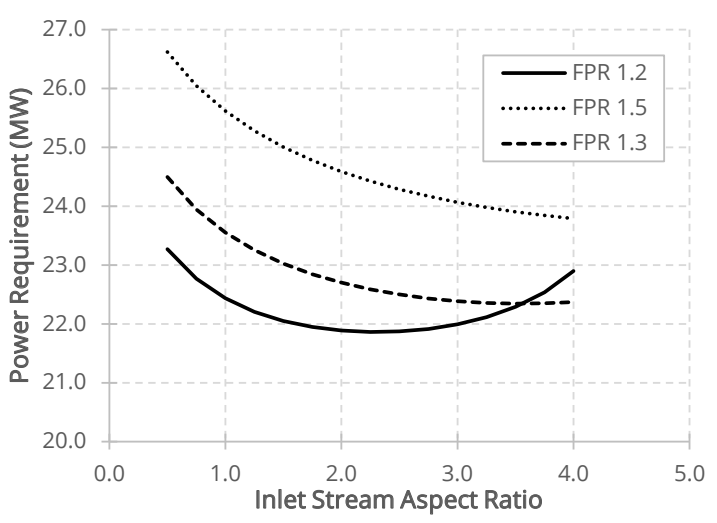

(b) Power requirement.

Figure 12: Power for arrays with varying aspect ratio of the inlet stream (neglecting nacelle drag).

a high inlet pressure loss case - such as might be seen for a high aspect ratio inlet transitioning to a circular fan face cross-section, or for an s-duct inlet - the optimum fan pressure ratio increases. This is compounded by the introduction of installation terms which further increases the optimum fan pressure ratio. The additional mass flow (and hence power) required from a low pressure ratio to compensate for either inlet pressure loss or the drag of a nacelle is noticeably greater than for a high pressure ratio fan. Hence minimising the fan pressure ratio is not always the ideal route to minimising the propulsor power requirement. The additional design variables introduced in the analyses demonstrated that the optimum design is obtained when the results of changes in $h / \delta$ and array length are balanced. The variables provide an additional degree of freedom which may be used to design each individual propulsor in order to optimise the array as a whole:

- Number of Propulsors

- Fan Pressure Ratio

- Inlet Aspect Ratio

- Net Propulsive Force

Each parameter has an impact on the array configuration and the performance of the individual propulsors. The method developed in this research enables the quick assessment of multiple configurations to assist in the preliminary assessment of each configuration and final selection of an optimised set of parameters. The presented results have been primarily in terms of the power saving coefficient, however, it should be noted that the optimum configuration from a power saving coefficient perspective may not be the optimum with regards to minimising power consumption (Figures 11 and 12).

Although previous research has shown that PSC can be improved by increasing the ingested drag of the array, it is not the only route to improvement of PSC. Simulations performed for the N3-X case have shown that the optimum FPR point with respect to PSC does not occur for a configuration with the highest percentage of ingested drag. Equally, a lower loss system will have improved power consumption, despite being a smaller array with a slightly lower ingested drag percentage. The results highlight the importance of tying the propulsion system and aircraft performance together at an early stage.

A limitation of the 1-D control volume method presented here is the inability to directly represent the additional intake loss incurred by ingesting the boundary layer and the losses in fan efficiency produce by flow distortion. Alternative methods are still required to identify the system-specific losses produced in the inlet.

Further research will extend the method to application for simulation at off-design conditions. Previous research on BLI system performance focuses almost exclusively on simulating design point performance, particularly with respect to the analytical methods. However, full assessments of aircraft making use of BLI system will require an off-design modelling procedure.

\section{Acknowledgements}

The authors would like to extend their gratitude to NASA for making this work possible under grant number NNX13AI78G. Many thanks go also to staff at Cranfield University for their advice and assistance. 


\section{References}

1 Flightglobal. Aircraft paint suppliers explore sharkskin coating. http://www.flightglobal.com/news/articles/aircraft-paint-suppliers-explore-sharkskin-coating381646/, 2013. Accessed: 2015-04-28.

2 Flightglobal. EasyJet turns to nanotechnology to save fuel. http://www.flightglobal.com/news/articles/easyjet-turns-to-nanotechnology-to-save-fuel-353148/, 2011. Accessed: 2015-04-28.

3 A M O Smith and Howard E Roberts. The Jet Airplane Utilizing Boundary Layer Air for Propulsion. Journal of the Aeronautical Sciences (Institute of the Aeronautical Sciences), 14(2):97-109, 1947. doi: $10.2514 / 8.1273$.

4 Leroy H. Smith. Wake ingestion propulsion benefit. Journal of Propulsion and Power, 9(1):74-82, 1993. ISSN 0748-4658. doi: 10.2514/3.11487.

5 A P Plas, M A Sargeant, V Madani, D Crichton, E M Greitzer, T P Hynes, and C a Hall. Performance of a Boundary Layer Ingesting (BLI) Propulsion System. In 45th AIAA Aerospace Sciences Meeting and Exhibit, 8 - 11 January 2007, Reno, NV, USA, number AIAA 2007-450, 2007. doi: 10.2514/6. 2007-450.

6 Arne Seitz, Julian Bijewitz, Sascha Kaiser, and Guido Wortmann. Conceptual investigation of a propulsive fuselage aircraft layout. Aircraft Engineering and Aerospace Technology, 86(6):464-472, 2014. doi: 10.1108/AEAT-06-2014-0079.

7 Susan Althoff Gorton, Lewis R Owens, Luther N Jenkins, Brian G Allan, and Ernest P Schuster. Active flow control on a boundary-layer-ingesting inlet. In 42nd AIAA Aerospace Sciences Meeting and Exhibit, Reno, NV, USA, number AIAA 2004-1203, 2004. doi: 10.2514/6.2004-1203.

8 Martin Rein, Stefan Koch, and Markus Rutten. Experimental Investigations on the Influence of Ingesting B.L. into a Diverterless S-Duct Intake. In 52nd Aerospace Sciences Meeting, National Harbor, MD, USA, number AIAA 2014-0373, 2014. doi: 10.2514/6.2014-0373.

9 David L Daggett, Ron Kawai, and Doug Friedman. Blended Wing Body Systems Studies: Boundary Layer Ingestion Inlets With Active Flow Control. Technical Report NASA/CR-2003-212670, NASA Langley Research Center, 2003.

10 James L Felder, Hyun Dae Kim, and Gerald V Brown. Turboelectric distributed propulsion engine cycle analysis for hybrid wing body aircraft. In 47th AIAA Aerospace Sciences Meeting, Orlando, FL, USA, number AIAA 2009-1132, 2009. doi: 10.2514/6.2009-1132.

11 J. I. Hileman, Z. S. Spakovszky, M. Drela, M. A. Sargeant, and A. Jones. Airframe Design for Silent Fuel-Efficient Aircraft. Journal of Aircraft, 47(3):956-969, 2010. ISSN 0021-8669. doi: 10.2514/1. 46545.

12 Jason Welstead and James L Felder. Conceptual Design of a Single-Aisle Turboelectric Commercial Transport with Fuselage Boundary Layer Ingestion. In 54th AIAA Aerospace Sciences Meeting, San Diego, CA, USA, number AIAA 2016-1027, 2016. doi: 10.2514/6.2016-1027.

13 Jl Felder and Hyun Dae Kim. Control Volume Analysis of Boundary Layer Ingesting Propulsion Systems With or Without Shock Wave Ahead of the Inlet. In 49th AIAA Aerospace Sciences Meeting, Orlando, FL, USA, number AIAA 2011-222, 2011. doi: 10.2514/6.2011-222.

14 Esteban A Valencia, Devaiah Nalianda, Panagiotis Laskaridis, and Riti Singh. Methodology to assess the performance of an aircraft concept with distributed propulsion and boundary layer ingestion using a parametric approach. Proceedings of the Institution of Mechanical Engineers, Part G: Journal of Aerospace Engineering, 229(4):682-693, 2015. doi: 10.1177/0954410014539291.

15 Chengyuan Liu, Daniel Ihiabe, Panagiotis Laskaridis, and Riti Singh. A preliminary method to estimate impacts of inlet flow distortion on boundary layer ingesting propulsion system design point performance. Proceedings of the Institution of Mechanical Engineers, Part G: Journal of Aerospace Engineering, 228(9):1528-1539, 2014. doi: 10.1177/0954410013496750. 
16 R. Chue, E. M. Greitzer, C. S. Tan, T. P. Hynes, and J. P. Longley. Calculations of Inlet Distortion Induced Compressor Flow Field Instability. International Journal of Heat and Fluid Flow, 10(3): 211-223, 1989. doi: 10.1016/0142-727X(89)90040-4.

17 Mark Drela. Power balance in aerodynamic flows. AIAA journal, 47(7):1761-1771, 2009. doi: 10. $2514 / 1.42409$.

18 Aurélien Arntz, Olivier Atinault, and Alain Merlen. Exergy-Based Formulation for Aircraft Aeropropulsive Performance Assessment: Theoretical Development. AIAA Journal, 53(6):1627-1639, 2014. doi: 10.2514/1.J053467.

19 Jonathan C Gladin, Brian K Kestner, Jeff S Schutte, and Dimitri N Mavris. Engine Design Strategy for Boundary Layer Ingesting Propulsion Systems With Multiple Non-Symmetric Engine Inlet Conditions. In ASME Turbo Expo 2013: Turbine Technical Conference and Exposition, San Antonio, TX, USA, number GT2013-95905, 2013. doi: 10.1115/GT2013-95905.

20 AGARD. Guide to In-Flight Thrust Measurement of Turbojets and Fan Engines. AGARD 237, 1979.

21 Larry W Hardin, Gregory Tillman, Om P Sharma, Jeffrey Berton, and David J Arend. Aircraft System Study of Boundary Layer Ingesting Propulsion. In 48th AIAA/ASME/SAE/ASEE Joint Propulsion Conference and Exhibit, Atlanta, GA, USA, number AIAA 2012-3993, 2012. doi: 10. 2514/6.2012-3993.

22 AP Plas. Performance of a boundary layer ingesting (BLI) propulsion system. Master's thesis, 2006.

23 Darrell Williams. Application of boundary layer theory to BLI simulation [Personal Communications], 2015.

24 J D Anderson. Fundamentals of Aerodynamics. McGraw-Hill Education, 1991. ISBN 0072373350. doi: 10.1371/journal.pcbi.1000716.

25 B. S. Stratford and G. S. Beavers. The Calculation of the Compressible Turbulent Boundary Layer in an Arbitrary Pressure Gradient-A Correlation of certain previous Methods. Technical Report ARC-3207, Aeronautical Research Council, 1961.

26 Iain G. Currie. Fundamental Mechanics of Fluids. CRC Press, 2002. ISBN 0824708865.

27 Hermann Schlichting. Boundary layer theory. part 2 - turbulent flows. Technical Report NACA-TM1218, Zentrale fuer Wissenschaftliches Berichtswesen, Berlin, Germany, 1949.

28 Herrmann Schlichting and Klaus Gersten. Boundary-layer theory. Springer, 2000. ISBN 3540662707.

29 Andrew Rolt and John Whurr. Optimizing Propulsive Efficiency in Aircraft with Boundary Layer Ingesting Distributed Propulsion. In 22nd International Symposium on Air Breathing Engines, 25 30 October 2015, Phoenix, AZ, USA, number ISABE-2015-20201, 2015.

30 Robert H Liebeck. Design of the blended wing body subsonic transport. Journal of aircraft, 41(1): 10-25, 2004. doi: 10.2514/1.9084.

31 Chana Goldberg, Devaiah Nalianda, and Riti Singh. Techno-economic and environmental risk assessment of a blended wing body with distributed propulsion. In 51st AIAA/SAE/ASEE Joint Propulsion Conference, Orlando, FL, USA, number AIAA 2015-4024, 2015. doi: 10.2514/6.2015-4024.

32 J Berton and W Haller. A noise and Emissions Assessment of the N3-X Transport. In 52nd Aerospace Sciences Meeting, National Harbor, MD, USA, number AIAA 2014-0594, 2014. doi: 10.2514/6. 2014-0594.

33 James Felder, Gerald Brown, Hyun Kim, and Julio Chu. Turboelectric Distributed Propulsion in a Hybrid Wing Body Aircraft. In 20th International Society for Airbreathing Engines, Gothenburg, Sweden, number ISABE-2011-1340, 2011.

34 James L Felder, Hyun Dae Kim, and Gerald V Brown. An Examination of the Effect of Boundary Layer Ingestion on Turboelectric Distributed Propulsion Systems. In 49th AIAA Aerospace Sciences Meeting, Orlando, FL, USA, number AIAA 2011-300, 2011. doi: 10.2514/6.2011-300. 
35 Arne Seitz and Corin Gologan. Parametric design studies for propulsive fuselage aircraft concepts. CEAS Aeronautical Journal, 6(1):69-82, 2015. doi: 10.1007/s13272-014-0130-3.

${ }^{36}$ Hyoungjin Kim and Meng-Sing Liou. Flow Simulation of N3-X Hybrid Wing-Body Configuration. In 51st AIAA Aerospace Sciences Meeting, Grapevine (Dallas/Ft. Worth Region), TX, USA, number AIAA 2012-221, 2013. doi: 10.2514/6.2013-221. 
2016-07-31

\section{Installed performance assessment of a} boundary layer ingesting distributed propulsion system at design point

\section{Goldberg, Chana}

American Institute of Aeronautics and Astronautics

Goldberg, C. et al. (2016) Installed performance assessment of a boundary layer ingesting distributed propulsion system at design point, 52nd AIAA/SAE/ASEE Joint Propulsion

Conference, Propulsion and Energy Forum, Salt Lake City, Utah, United States of America, 25-27 July

http://dx.doi.org/10.2514/6.2016-4800

Downloaded from Cranfield Library Services E-Repository 